\title{
Archéopages
}

Archéopages

Archéologie et société

43 | 2016

Médecines

\section{Plantes médicinales et régime alimentaire}

Les nonnes de Clairefontaine au XVIII ${ }^{\mathrm{e}}$ siècle

Medical plants and diet. The nuns of Clairefontaine in the $18^{\text {th }}$ century

Plantas medicinales y régimen alimenticio. Las monjas de Clairefontaine en el siglo XVIII

Sidonie Preiss

\section{(2) OpenEdition}

1 Journals

Édition électronique

URL : https://journals.openedition.org/archeopages/2056

DOI : 10.4000/archeopages.2056

ISSN : 2269-9872

Éditeur

INRAP - Institut national de recherches archéologiques préventives

Édition imprimée

Date de publication : 1 novembre 2016

Pagination : 52-59

ISSN : $1622-8545$

\section{Référence électronique}

Sidonie Preiss, «Plantes médicinales et régime alimentaire », Archéopages [En ligne], 43 | 2016, mis en ligne le 01 décembre 2018, consulté le 03 juin 2021. URL : http://journals.openedition.org/ archeopages/2056 ; DOI : https://doi.org/10.4000/archeopages.2056 


\section{Plantes médicinales et régime alimentaire Les nonnes de Clairefontaine au XVIIII ${ }^{\mathrm{e}}$ siècle}

Sidonie Preiss Institut royal des Sciences naturelles de Belgique
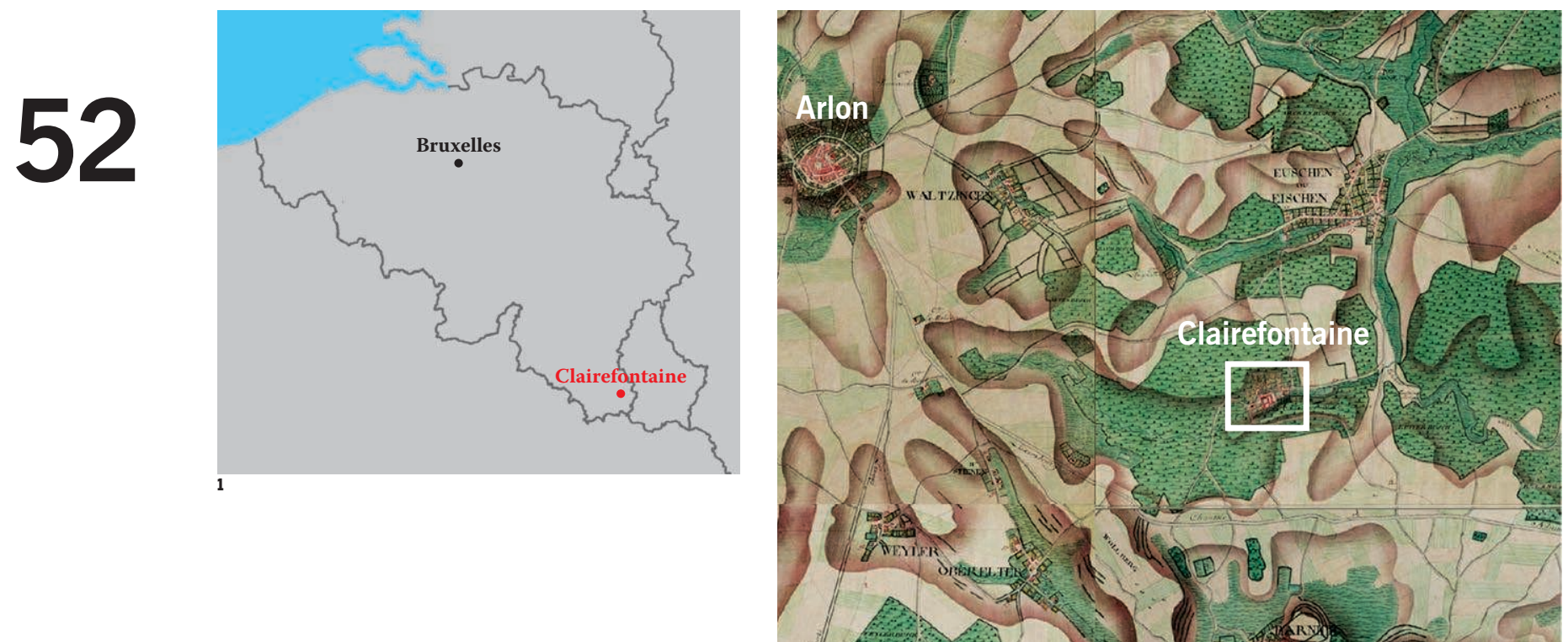
Les religieuses de l'époque moderne ont longtemps été considérées comme des femmes pieuses, détachées du monde séculier, ayant choisi une vie stricte, empreinte de discipline. Dans cette perspective, rejoindre le couvent devait impliquer des changements radicaux du mode de vie des jeunes filles, notamment pour celles issues de la haute société, forcées d'abandonner leurs biens matériels et leurs habitudes nobles. Cependant, cette vision traditionnelle a été nuancée par des recherches récentes qui ont démontré que de multiples aspects de la vie mondaine étaient transposés dans la vie conventuelle (Johnson et Matthews-Grieco, 1997 ; Hamburger et Suckale, 2008 ; Signori, 2008).

À la suite des fouilles menées à l'abbaye cistercienne de Clairefontaine [ill. 1], des travaux interdisciplinaires effectués par une partie de l'Unité d'Archéosciences de l'Institut royal des Sciences naturelles de Belgique (analyses archéobotaniques ${ }^{\mathbf{1}}$ et archéozoologiques ${ }^{\mathbf{2}}$ ) ont permis de reconstituer quelques aspects du quotidien de la dernière génération de nonnes ayant occupé l'abbaye jusqu'en 1794. À cette époque, l'abbaye de Clairefontaine - fondée vers le milieu du XIII ${ }^{\mathrm{e}}$ siècle par les comtes de Luxembourg afin d'y installer leur nécropole dynastique - était l'une des plus prestigieuses de la région et n'acceptait que les filles de haute naissance, principalement issues de la noblesse luxembourgeoise (Goffinet, 1877, 1907). Malgré le démantèlement d'une grande partie des bâtiments pendant la Révolution française, la fouille du site ${ }^{\mathbf{3}}$ a mis au jour les latrines du couvent, datées du XVIII ${ }^{\mathrm{e}}$ siècle (Herremans et Coomans, 2013 ; Herremans, 2013). En plus d'une grande quantité d'objets, elles contenaient de très nombreux restes botaniques et fauniques. Les restes fauniques et les artefacts ont été récoltés à vue lors de la fouille et des échantillons de sédiments ont été prélevés dans chaque couche, puis traités et tamisés en laboratoire en vue d'analyses archéobotaniques et archéozoologiques.
Une alimentation riche et variée composée de nombreuses friandises

L'étude des objets retrouvés en fouille de même que celle des sources écrites révèlent certains aspects de la vie entre les murs du couvent. L'analyse ${ }^{\mathbf{4}}$ des comptes de l'abbaye entre novembre 1742 et octobre 1743 (Herremans, 2013 ; Herremans et De Clercq, 2013) montre ainsi que du thé et du chocolat étaient achetés régulièrement et que les sœurs de Clairefontaine aimaient leur thé sucré et accompagné de pâtisseries, comme il était de coutume pour les dames nobles de cette époque. Ces produits étaient consommés en groupe comme le suggère la découverte d'une variété de services à thé ou à chocolat en céramique de haute qualité. Toutefois, à côté de ces services haut de gamme, des ustensiles et récipients de moins bonne facture étaient aussi importés. Signalons encore que les nonnes consommaient des spiritueux tels que de l'eaude-vie et de l'hydromel. Plusieurs petits verres à liqueurs décorés à la façon de Bohème renvoient à la consommation de ces boissons (Herremans et al., 2013).

L'alimentation d'origine animale (Goffette, 2013) était de qualité, comme en témoigne la présence d'animaux nobles tels que le cygne, de gibier à plumes et à poils et la sélection de jeunes animaux domestiques, notamment le veau, qui fournissaient une viande tendre. La viande grasse de mammifères a régulièrement été servie à table : les principales espèces consommées sont les classiques bœufs, porcs et moutons, élevés dans le domaine et les dépendances de l'abbaye. Le porc est majoritaire en nombre de restes, devant les caprinés et le bœuf. Cette prépondérance du porc est rare en milieu religieux. L'environnement fortement boisé de l'abbaye, utilisé pour la glandée, a dû favoriser son élevage. Les poissons, les écrevisses et les cuisses de grenouilles ainsi qu'une importante variété de viandes de volaille participaient à la diversité des mets consommés. Toutes les espèces consommées ont pu être élevées ou prélevées dans l'environnement direct de l'abbaye, à l'exception des poissons marins importés sous forme préparée.

Les analyses des restes botaniques ont porté sur deux volets principaux : les macrorestes végétaux, c'est-à-dire les fruits et les graines (étudiés par la carpologie) et les microrestes végétaux représentés par les grains de pollen et les spores (étudiés par la palynologie) (Preiss et Court-Picon, 2013). Nous nous intéressons ici principalement aux résultats carpologiques. Ils sont marqués par une forte densité de restes dans les prélèvements et une grande diversité taxonomique, et ce malgré une conservation variable ${ }^{\mathbf{5}}$. La majorité des taxons identifiés appartiennent à des plantes sauvages, mais, en ce qui concerne les quantités, les plantes cultivées et/ou cueillies dominent largement le corpus [ill. 2]. L'étude carpologique montre que les restes de fruits sont non seulement très

\footnotetext{
À partir de ces recherches associées aux données archéologiques et historiques, il transparaît clairement que les sœurs de Clairefontaine ont adopté dans le monastère un mode de vie conforme à leur origine sociale. Tout comme dans le monde séculier, l'apparence et le style de vie semblent avoir été leur priorité, au lieu de la discipline monastique. Dans cet article, nous nous intéressons tout particulièrement aux habitudes alimentaires de ces nonnes, en particulier par le biais des analyses carpologiques (études des graines et des fruits retrouvés en fouille). Ces différentes recherches soulignent en effet que l'alimentation de la dernière génération des nonnes de Clairefontaine était fort riche en aliments gras et sucrés, tandis que la présence notable de plantes aux vertus médicinales communes suggère qu'elles ont pu utiliser ces dernières pour alléger ce régime et pallier ses désagréments.

Davy Herremans.

l'historienne Isabell

5. Les restes principalement

carbonisés et minéralisés.

préservation n'est

n'a pas toujours permi

d'identifier les restes

au niveau de l'espèce.

Aurélie Salavert

Mona Court-Picon

et Sidonie Preiss

2. Effectuées par

in Goffette.
} 


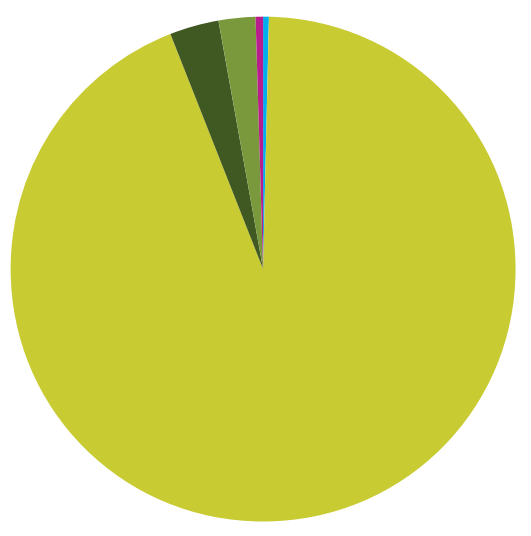

$\mathrm{NMI}=10950$

Volume $=1 \mathrm{~L}$

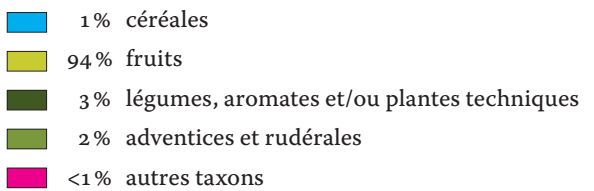

3

$\mathrm{NMI}=344.5 \quad$ Taxa (nom scientifique des plantes)

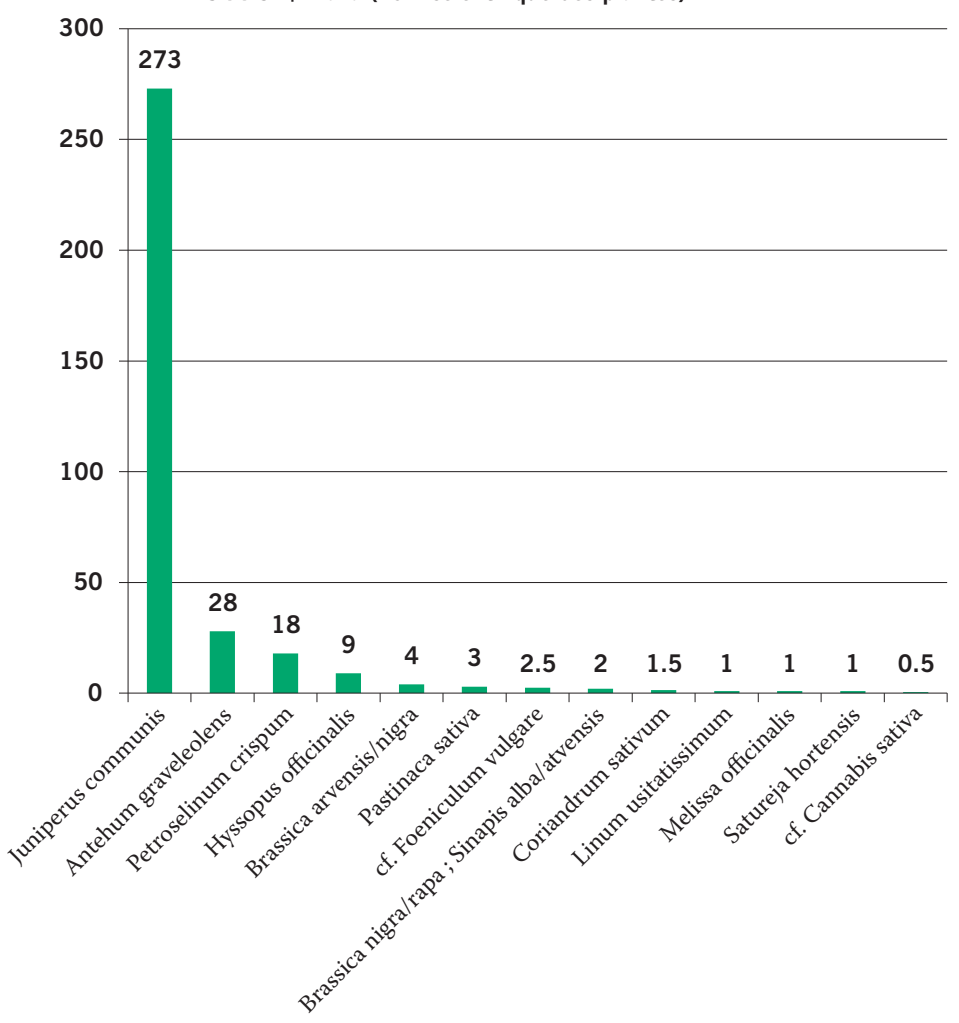

6. Plantes liées à l'artisanat, qui

demandent un savoir-

faire et un protocole

d'extraction bien précis

pour transformer

la matière première

en produit fini. Il s'agit

principalement de

plantes textiles et/ou

oléagineuses, comme

le lin et le chanvre.

7. Une plante aux vertus

diurétiques est une

plante possédant

des substances qui

augmentent la sécrétion

et la production d'urine.

8. Eau-de-vie à base de

céréales (principalement

à base d'orge et de seigle)

aromatisée à partir

de baies de genévrier

commun. abondants ( $94 \%$ du total), mais aussi très diversifiés avec 17 taxons différents. Parmi eux, la fraise, la framboise et la mûre comptent pour la majeure partie. Le sureau noir, le cassis et la groseille sont également bien représentés. La noisette, différentes variétés de prunes, de cerises et le raisin occupent une plus petite part. On note aussi la présence de la figue, de la nèfle, de la pomme et/ou de la poire, de la cornouille et peut-être de la noix. La dominance des petits fruits des bois, présents dans l'environnement du voisinage de l'abbaye, suggère une collecte locale. La comptabilité du milieu du XVIII ${ }^{\mathrm{e}}$ siècle apporte un complément d'information au sujet de la consommation des fruits. En effet, en plus des espèces présentes dans les vestiges carpologiques, elle mentionne les agrumes tels que les citrons et les oranges, les amandes et les châtaignes. La présence de légumes, d'aromates et/ou des plantes techniques ${ }^{\mathbf{6}}$ dans les résultats carpologiques est remarquable. Peu abondants, ces restes sont en revanche très diversifiés, avec près de 13 taxons identifiés [ill. 3].
L'information la plus marquante réside dans la quantité exceptionnelle de baies de genévrier et dans la diversité des plantes aux vertus digestives mises en évidence par l'étude carpologique. Plusieurs types de restes de genévrier sont représentés : majoritairement des graines, mais également des fruits carbonisés et imbibés et de nombreux grains résinifères. Aucun grain de pollen, ni charbon de bois de cette espèce n'a été retrouvé (Preiss et Court-Picon, 2013). Ceci suggère d'une part que les baies de genévrier commun ont été intentionnellement récoltées et qu'elles ne viennent pas de l'environnement direct de l'abbaye et, d'autre part, qu'elles ont été utilisées dans un but particulier. Les baies et les graines de genévrier commun ont pu être utilisées pour la préparation de spiritueux, pour l'encens ou encore pour leurs qualités diurétiques ${ }^{7}$ et digestives. Aucune mention de genévrier n'est enregistrée dans les comptes, ni même de genièvre ${ }^{\mathbf{8}}$. Cependant, il est fait mention d'achat d'eau-de-vie pour la communauté, sans précision sur les quantités. Cette eau-de-vie pourrait avoir été 


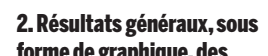

forme de graphique, des

différents types de restes,

classés par catégories

et retrouvés dans les latrines

du xvIII' siècle de l'abbaye

de Clairefontaine.

NMI : nombre minimum

d'individu(s), L: litre.

La catégorie "| fruits »

regroupe des fruits cueillis

et/ou cultivés, celle

nommée "I autres taxons "),

l'ensemble des taxons

ayant été identifiés au seul

rang de genre ou de famille.

3. Légumes, aromates

et/ou plantes techniques

retrouvés dans les latrines

du xvill' siècle de l'abbaye

de Clairefontaine. cf.: confer,

NMI : nombre minimum

d'individus.
9. Le méteil est

traditionnellement

un mélange à proportion

égale de froment et

de seigle, et, plus

généralement, un

mélange de diverses

céréales ou de céréales

et de légumineuses.

10. Plantes qui poussent

spontanément à

proximité des lieux

habités par les êtres

humains. utilisée, en partie, pour la confection de genièvre. En outre, de nombreuses autres herbes aromatiques mises en évidence par l'étude, comme l'aneth, le fenouil et la coriandre, ont également pu être appréciées pour leurs vertus digestives. Le persil et l'hysope ont pu servir à la fois à l'assaisonnement des plats et à favoriser la digestion.

Les sources écrites mentionnent l'artichaut et le céleri, dont des plants ont été achetés pour le jardin, ou encore des pommes de terre acquises pour les besoins de la communauté. Concernant les aromates, on note surtout l'achat de moutarde sous forme de farine ou de graines. Le houblon est très souvent mentionné : provenant des jardins de l'abbaye, il est vendu ou bien utilisé pour la confection de la bière de la communauté.

Les plantes textiles et oléagineuses telles que les moutardes, le chanvre et le lin sont pratiquement absentes des assemblages carpologique et palynologique (Preiss et Court-Picon, 2013). Le lin est mentionné en recettes et en dépenses dans les livres de comptes. Le chanvre l'est uniquement en recettes : il provient de la ferme de la basse-cour de l'abbaye. Des toiles de chanvre sont vendues par la communauté.

Les céréales sont très peu nombreuses dans notre corpus, ce qui est courant en contexte de latrines. Elles sont principalement représentées par le seigle et le millet. Les macrorestes sont en plus grande partie enregistrés sous forme de glumes et de téguments, suggérant une transformation importante des grains (pour les préparations culinaires de type gruau, porridge, pain, galettes, pâtisseries, etc.). Les mentions de céréales dans les sources historiques contrastent avec la quantité de restes retrouvée dans les latrines. En effet, c'est la catégorie des céréales qui est le plus souvent mentionnée et uniquement en recettes. Elles sont la production des dépendances de l'abbaye. Il est fait en effet mention d'avoine et de méteil ${ }^{\mathbf{9}}$, de seigle, de froment et d'orge mondé, ce qui signifie la présence de l'orge vêtue. Bien entendu, les céréales étaient consommées par les nonnes de Clairefontaine, mais leur transformation ne se faisait certainement pas directement dans les cuisines de la communauté, ce qui explique la discrétion des céréales dans les spectres archéobotaniques. Les légumineuses cultivées, étonnamment absentes des restes carpologiques, ont été identifiées par la palynologie avec principalement la lentille, mais aussi la fève et/ou le pois, ce qui suggère là encore une transformation importante des produits (Preiss et Court-Picon, 2013). On retrouve seulement deux mentions de légumineuses dans les sources écrites étudiées : des fèves pour engraisser les porcs, certainement en complément de la glandée en fonction des périodes de l'année, et des petites fèves utilisées pour la basse-cour, soit comme fourrage pour les animaux, soit comme semences.

Très peu d'espèces exotiques et importées ont été identifiées dans les assemblages carpologiques et palynologiques. On enregistre un fruit de chardon béni, plante ornementale et/ou médicinale, et des akènes de figues. Dans les inventaires de comptabilité, on retrouve de nombreuses espèces exotiques : des fruits, comme les olives, les figues, les citrons et oranges, les amandes sont achetés. Des épices comme le gingembre, le poivre et la cannelle sont également souvent mentionnées. Ces épices, utilisées par l'aristocratie entre le $\mathrm{XIV}^{\mathrm{e}}$ et le XVI ${ }^{\mathrm{e}}$ siècle, sont devenues beaucoup plus populaires au $\mathrm{XVIII}^{\mathrm{e}}$ siècle. Elles ne révèlent donc pas nécessairement le statut noble des nonnes de Clairefontaine. En revanche, l'achat de thé et de chocolat souligne quant à lui un haut rang social. Ces boissons dites « exotiques », introduites en Europe dans le courant du XVII ${ }^{\mathrm{e}}$ siècle, ont fait partie intégrante des sociabilités de l'aristocratie et de la haute bourgeoisie. Le coût de ces produits importés au XVII ${ }^{\mathrm{e}}$ et XVIII ${ }^{\mathrm{e}}$ siècle, tout comme celui du café, classe ces denrées parmi les produits luxueux. Cela ajoute à leur consommation l'image affichée du prestige.

Les plantes sauvages sont très diversifiées et appartiennent principalement à la flore adventice des cultures, mais aussi aux rudérales ${ }^{\mathbf{1 0}}$ liées aux occupations humaines, aux habitations, aux zones riches en nitrates, etc. Un certain nombre d'entre elles ont des vertus médicinales, comme l'armoise, la fumeterre ou le plantain lancéolé.

\section{De nombreuses plantes aux vertus médicinales communes}

Dans la comptabilité de la communauté, il n'est fait aucune mention de plantes médicinales ou encore de plantes sauvages. En archéobotanique, il n'est pas aisé de faire un lien direct et logique entre la présence des plantes, leur utilisation et leurs vertus médicinales. En effet, de très nombreuses plantes, cultivées ou sauvages, ont des propriétés médicinales. Elles ne sont pas toujours utilisées volontairement dans le but de soigner ou de guérir, mais parfois uniquement en tant qu'aromate ou condiment. Cependant, à

Clairefontaine, les résultats carpologiques mettant en évidence la présence importante de plantes ayant comme propriétés de faciliter la digestion et de nettoyer les toxines du corps, nous avons émis l'hypothèse que cette association n'était pas liée uniquement au hasard de la préservation du corpus archéobotanique. En effet, il pourrait y avoir un lien entre la consommation d'une alimentation riche en viande, en graisse d'origine animale et végétale et en sucres présentée brièvement ci-dessus et celle de plantes aux vertus digestives, diurétiques et dépuratives. Les propriétés d'une dizaine de plantes identifiées dans le corpus des latrines de Clairefontaine ont ainsi pu être précisées à partir de divers ouvrages spécialisés sur le sujet (Dodoens et L'Escluse, 1557 ; Boisvert, 2003 ; Allen et Hatfield, 2004) : certaines cultivées comme le chardon béni, l'aneth, 


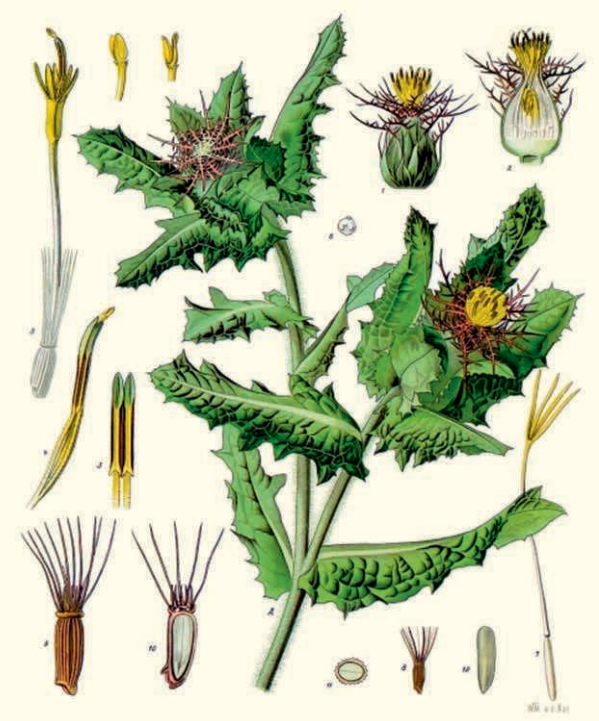

டீ
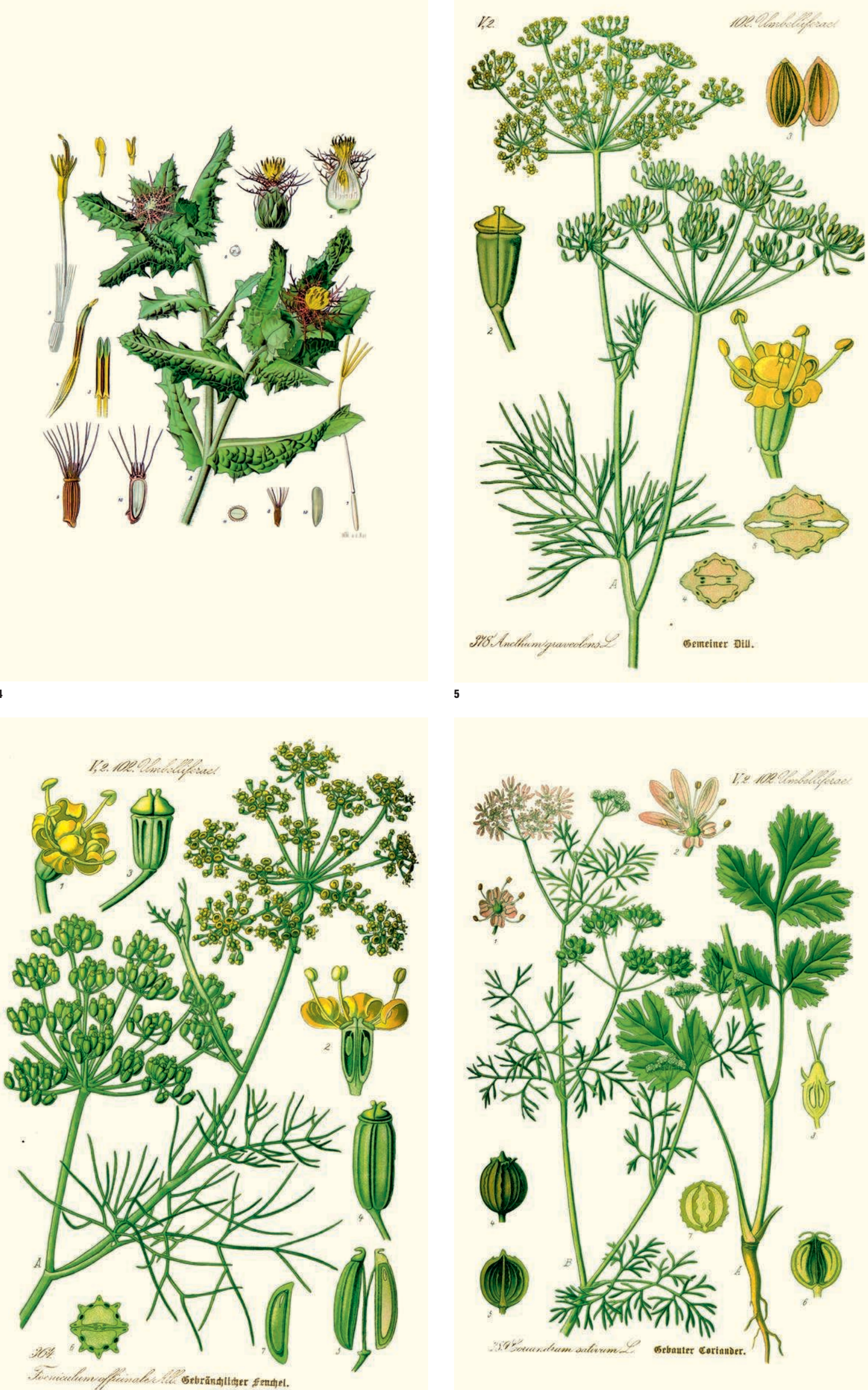

4. Chardon béni, Centaurea benedicta L.: c'est un

détoxifiant du foie. II peut être utilisé pour ses propriétés

digestives et stomachiques,

contre les infections ainsi que

les piqûres d'insectes.

5. Aneth, Anethum graveolens L. : selon les préparations

faites à partir des différentes parties de la plante, elle peut aider à arrêter les

vomissements et les maux

de ventre, est diurétique,

digestive et carminative.

Elle peut calmer les

saignements menstruels.

6. Fenouil, Foeniculum vulgare

Mill.: toutes les parties de la

plante présentent des vertus

médicinales. Le fenouil est

diurétique et carminatif.

II allège la douleur des reins

et permet de dissoudre ou

de prévenir les calculs rénaux.

II apaise les douleurs

d'estomac et les nausées.

II est très bon pour les

poumons, la vue, le foie,

les reins, le transit intestinal

et est vermifuge.

7.Coriandre, Coriandrum

sativum L.: la graine de

coriandre préparée, prise

simplement ou enrobée

de sucre, consommée après

le repas, permet la satiété

et empêche l'inflammation

de l'œesophage. Elle stoppe

les vomissements et aide

à digérer la viande. Les

graines de coriandre sont

stimulantes, digestives

et carminatives. La coriandre

est recommandée pour

relever le tonus et après un

bon repas pour combattre

l'endormissement qui lui

succède. La coriandre est un

bon vermifuge et bactéricide

pouvant être employé lors

de dysenteries, diarrhées et

d'intoxications intestinales.

8. Persil, Petroselinum

crispum Mill. : en tant que

diurétique, permet de soigner

les affections des voies

urinaires. Le persil est

également un régulateur très

efficace des menstruations et

apaise les douleurs qui leurs

sont liées. Il est aussi un

remède contre l'épilepsie

et contre l'asthme et la toux. 


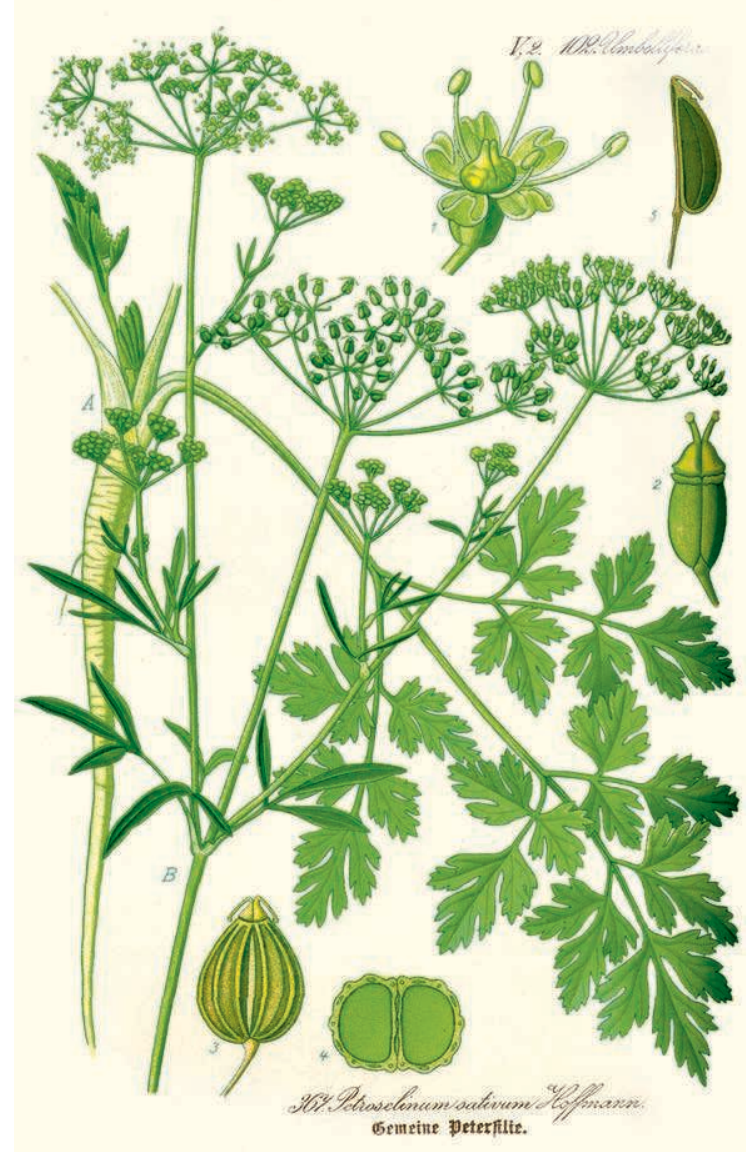

8 le fenouil, la coriandre, le persil et l'hysope, d'autres sauvages et considérées soit en tant qu'aromate comme le genévrier commun ou en tant que plantes sauvages comme l'armoise commune, la fumeterre officinale et le plantain lancéolé [ill. 4 à 13].

Bon nombre de ces plantes possèdent des vertus digestives (chardon béni, aneth, fenouil commun, coriandre, armoise commune et fumeterre officinale) et diurétiques (aneth, fenouil, persil, genévrier commun, armoise commune et fumeterre officinale), mais également carminatives, c'est-à-dire facilitant l'expulsion des gaz intestinaux (aneth, fenouil, coriandre et genévrier commun). De manière générale, elles aident à purifier le corps des toxines grâce à leurs effets régulateurs sur les fonctions de l'estomac (chardon béni, fenouil, genévrier, armoise commune et plantain lancéolé) et l'écoulement de la bile (fumeterre officinale), ou encore leurs pouvoirs dépuratifs pour les reins et le foie (fenouil, genévrier commun, fumeterre officinale, plantain lancéolé). En plus de ces nombreuses vertus en lien étroit avec le régime alimentaire, un grand nombre d'espèces citées ont des qualités vermifuges (fenouil, coriandre, hysope, genévrier commun, armoise commune), anti-infectieuses (chardon béni, hysope et genévrier commun), antifongiques (armoise commune) et antibactériennes (coriandre et armoise commune). Certaines apaisent les diarrhées et les dysenteries (coriandre et plantain lancéolé) et luttent contre les vomissements (aneth et coriandre) et les nausées (fenouil, plantain lancéolé). Enfin, une partie d'entre elles possèdent en plus la capacité de réguler les menstruations (aneth, persil, genévrier commun, armoise commune et plantain lancéolé).

L'ensemble des résultats ainsi obtenus pour les latrines du XVIII ${ }^{\mathrm{e}}$ siècle à Clairefontaine donne un bel aperçu de la culture matérielle et des préférences alimentaires de la dernière génération des sœurs de l'abbaye. Tout d'abord, si la majorité des ressources animales et végétales proviennent du domaine monastique et des zones environnantes, l'étude de la faune montre la présence d'espèces inhabituelles et une nourriture noble. Ensuite, bien que les restes botaniques ne révèlent pas particulièrement une consommation liée à un haut niveau social, les fruits et condiments sont très diversifiés et certains produits locaux ont pu être incorporés dans des préparations alimentaires plus élaborées. De plus, la présence remarquée de nombreuses espèces aux vertus digestives et médicinales peut être facilement associée au régime alimentaire décrit, riche en nutriments : beaucoup de protéines, de sucres et de pâtisseries. En plus d'avoir des vertus digestives détoxifiantes et anti-infectieuses, bon nombre de ces plantes possèdent des qualités pour améliorer les désagréments liées aux menstruations, donc 

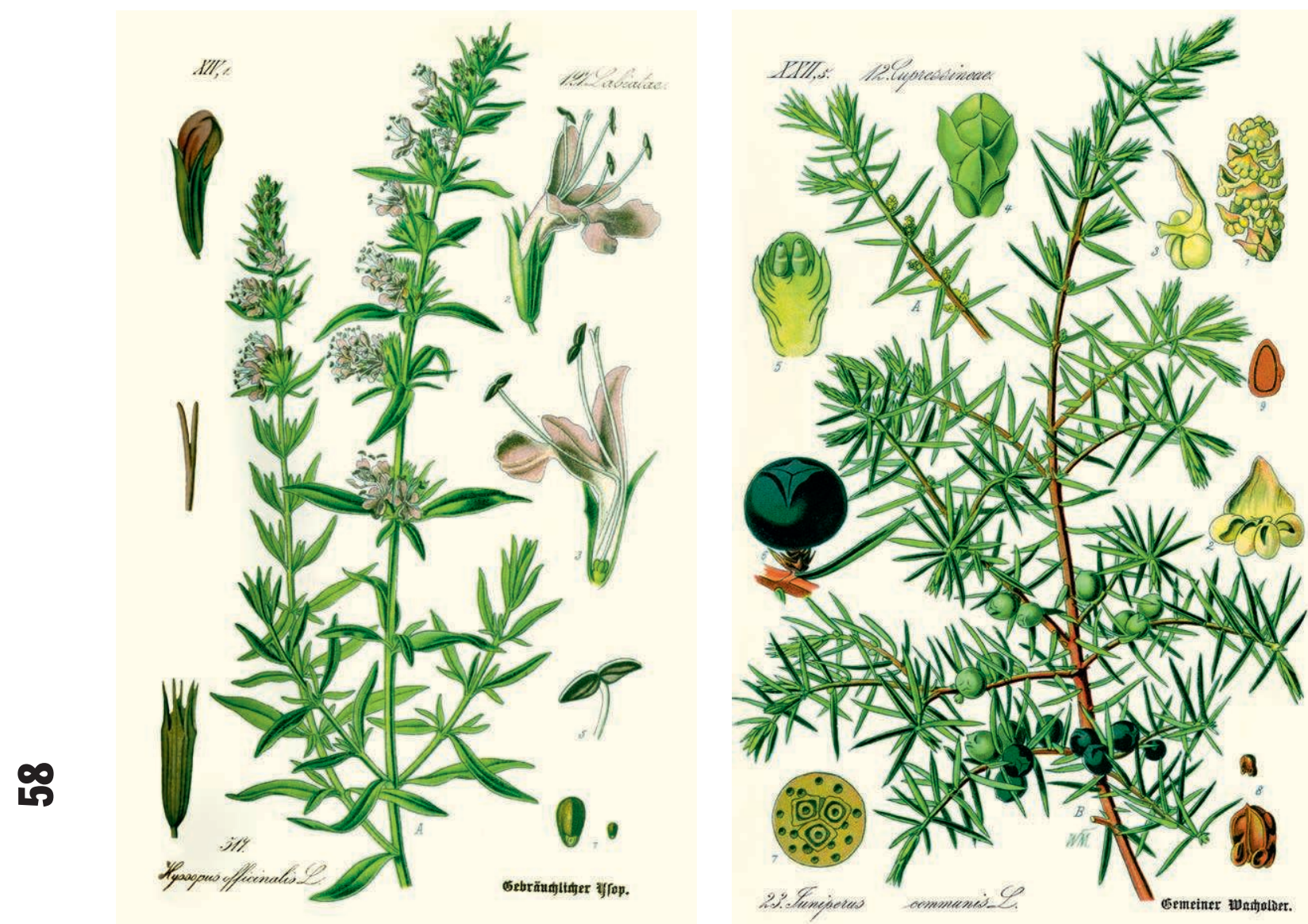

9. Hysope, Hyssopus

officinalis L: : selon les

préparations, l'hysope aide

dans les cas d'obstruction

de la poitrine, de souffle court

ou de toux difficiles à soigner.

Elle peut être un bon

vermifuge. Elle apaise

les douleurs de dents et,

en gargarisme, permet

de soigner les aphtes

et les infections diverses

de la muqueuse buccale.

10. Genévrier commun,

Juniperus communis L.: fruit

utile au bon fonctionnement

del'estomac, des poumons,

du foie et des reins. Il est

carminatif et diurétique.

Le jus des feuilles est

antivenimeux. La fumée

du fruit ou des feuilles brûlés

sert de répulsif et est anti-

infectieux. Le genévrier sert

de vermifuge. Il permet

également de réguler les flux

menstruels.

11. Armoise commune,

Artemisia vulgaris $\mathrm{L}$.

elle facilite la digestion, est

diurétique et aide à dissoudre

les calculs rénaux. Elle est

également un vermifuge

efficace. Elle restaure les flux

menstruels. Elle est

antibactérienne et

antifongique. Elle guérit les

maux d'estomac, est très

bonne pour calmer les nerfs

et soigne les règles diffficiles

et les troubles liés à la

ménopause.

12. Fumeterre officinale,

Fumaria officinalis L.:

elle a des vertus dépuratives

sur les reins, la vésicule

biliaire et le foie. Elle facilite

la digestion. La décoction

de fumeterre est diurétique.

Le jus de fumeterre mis

dans les yeux aiguise la vue

et l'éclaircit. Elle est un

remarquable régulateur

de l'écoulement biliaire.

Cependant, son utilisation

ne doit pas se faire sur des

périodes trop prolongées.

13. Plantain lancéolé,

Plantago lanceolata L.:

les feuilles confortent

l'estomac et calment les

nausées. La décoction

des feuilles arrête les flux

dysentériques et calme

les règles trop abondantes.

Les racines et les semences

sont très bonnes pour

la santé du foie et des reins.

Le plantain était utilisé

autrefois contre la jaunisse

et les ulcères des reins

et de la vessie. II a des vertus

anti-inflammatoires,

coagulante, antiseptique

et tonifiante. II peut servir

contre l'inflammation

des oreilles et des yeux.

Le jus de plantain en

décoction soigne la muqueuse

buccale, la douleur des

dents et le saignement

des gencives. 


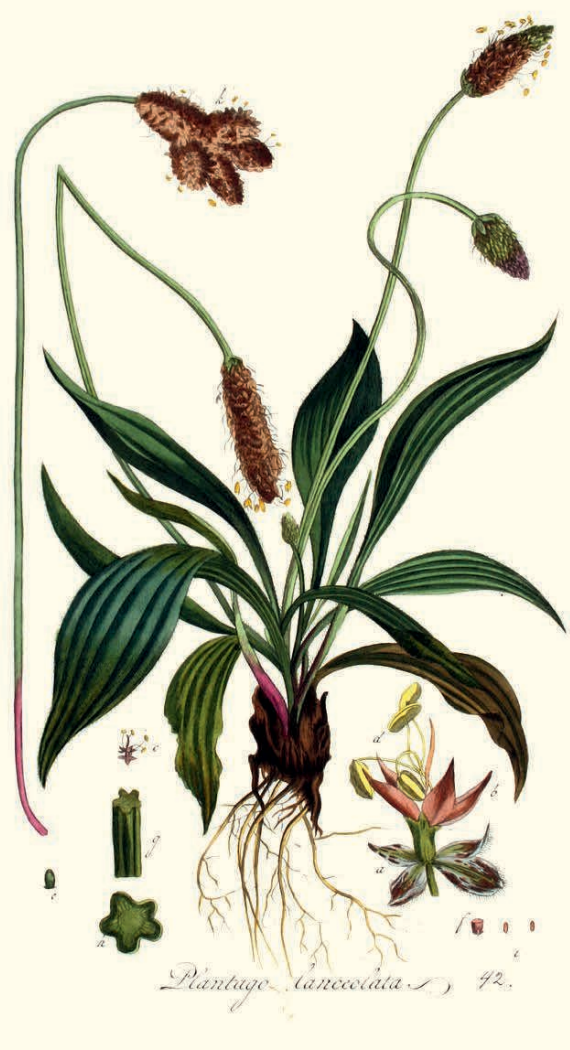

véritablement spécifiques pour les soins apportées aux jeunes femmes et aux femmes en général qui habitaient le couvent.

En confrontant l'ensemble des données obtenues, on peut déduire que la diète des sœurs était variée et plutôt calorique, mais traditionnelle et tout à fait en accord avec les pratiques de consommation de l'élite rurale de l'époque et qu'elles ont pu avoir recours aux plantes médicinales pour alléger ce régime trop riche en graisses animales et végétales. Si l'on ignore de quelles connaissances les nonnes de Clairefontaine disposaient sur les plantes médicinales, on sait que chaque abbaye s'est dotée d'un jardin des simples dès la période médiévale, à l'image du plan de l'abbaye de Saint-Gall qui figure trois jardins dont un jardin des simples. Pour appuyer notre hypothèse, il nous faudrait pouvoir comparer d'autres résultats semblables et proches pour cette même période. En attendant, la prudence reste donc de mise quant à l'hypothèse de l'utilisation volontaire de toutes ces plantes pour leurs vertus médicinales.

\section{Références bibliographiques}

Dodoens R., L'EsCluse C. DE, 1557, Histoire des plantes, Anvers, impr. de J. Loe, fac-similé 1978, Opsomer J.-E. (dir.), Bruxelles, Centre National d'Histoire des Sciences, 584 p.

Allen D. E., Hatfield G., 2004, Medicinal PLants in Folk Tradition: an Ethnobotany of Britain E Ireland, Portland, Cambridge, Timber Press, $431 \mathrm{p}$.

Boisvert C., 2003, Plantes et remèdes naturels, Genève, Aubanel, $527 \mathrm{p}$.

GoffetTe Q., 2013, Rapport préliminaire archéozoologique de l'abbaye de Clairefontaine, SPW-IRSNB, $75 \mathrm{p}$.

Goffinet H., 1877, Cartulaire de Clairefontaine. Recueil de documents presque tous inédits concernant cette ancienne abbaye, Arlon, P.-A. Brück, 284 p.

GofFINET H., 1907, « L'ancienne abbaye de Clairefontaine. Notice historique », Annales de l'Institut archéologique du Luxembourg, 42, p. 117-156.

Hamburger J. F., Suckale S., 2008, « Between Thi World and the Next: The Art of Religious Women in the Middle Age », in Hamburger J. F., Marti S. (éd.), Crown and veil. Female monasticism from the fifth to the fifteenth centuries, New York, Columbia University Press, p. 76-108.

Herremans D., 2013, Holy vows, worldly manners: Monastic space, consumption practices and social identity in the Cistercian nunnery of Clairefontaine, Zelzate, Ghent University Press, 1 vol., 275 p.
Herremans D., Coomans T., 2013, «L'abbaye de moniales cisterciennes de Clairefontaine (Luxembourg). Synthèse archéo-historique des quatre phases de construction, $\mathrm{XIII}^{\mathrm{e}}-\mathrm{XVIII}{ }^{\mathrm{e}}$ siècle », Bulletin Monumental, 171-2, p. 117-139.

Herremans D., De ClercQ W., 2013, « De herrinering blijft. Memoria en materiële cultuur in de monastieke ruimte van Clairefontaine (B)», Volkskunde. Tijdschrift over de cultuur van het dagelijkse leven 113-3, p. 282-305.

Herremans D., Cagno S., Vincke A., Janssens K., DE ClerCQ W., 2013, « All Crystal Clear: 18th-Century Glass à la façon de Bohème from the Cistercian Nunnery of Clairefontaine, Belgium ", Journal of Glass Studies, 55, p. 137-151.

Johnson G. A., Matthews-Grieco S.F., 1997, Picturing women in Renaissance and Baroque Italy, Cambridge, Cambridge University Press, 335 p.

Preiss S., Court-Picon M., 2013, Rapport détudes carpologique et palynologique de l'abbaye cistercienne de Clairefontaine, les latrines (XVIII ${ }^{e}$ s.), SPW-IRSNB, $14 \mathrm{p}$.

SIGNORI G., 2008, « Wanderers Between Worlds: Visitors, Letters, Wills, and Gifts as Means of Communication in Exhanges Between Cloister and the World », in Hamburger J. F., Marti S. (éd.), Crown and veil. Female monasticism from the fifth to the fifteenth centuries, New York, Columbia University Press, p. 259-274. 